\title{
Digitisers: their use in the entry of orders, urinalysis results, and isoenzyme interpretation in a clinical biochemistry computer system
}

\author{
JANE F LOUGHLIN, JAMES F TUCKERMAN, A RALPH HENDERSON \\ From University Hospital (University of Western Ontario) London, Ontario, Canada
}

SUMMARY The processes of order entry, urinalysis result, and isoenzyme interpretation entry into a laboratory computer system is a time consuming activity. We have designed a series of forms for use with a digitising pad that allow us rapidly to enter orders, urinalysis results, and isoenzyme interpretative comments into our laboratory computer system. We have shown that digitiser entry is always significantly faster than manual entry. Although there are many devices available to facilitate computer entry, we believe that the digitiser technique is an attractive option because of its ease of use, speed, reliability, and low cost.

It is generally accepted that a computer system in a hospital laboratory increases the overall productivity of the laboratory staff, although there are many operator-computer interactions that are, or may be extremely time consuming and prone to error. A major problem in any chemistry laboratory is passing data from the analyser to the computer; this is usually circumvented by direct interfacing of these devices. The only human intervention then required is instructing the computer to accept, process, or edit the stream of data from the analyser. In our experience the next most time consuming interaction is entering test requests into the laboratory computer. These are usually entered by means of the keyboard of the video display terminal. Test names are usually replaced by related short mnemonics to facilitate entry. Thus an SMA-12 request would have a mnemonic S12 and so on. Mnemonic entry certainly speeds up the process of test entry, but the large test repertoire of most teaching hospital laboratories requires a wide range of mnemonics. These are sometimes difficult to remember and new staff require considerable training before they are confident with these codes. None the less, mnemonics can still take a considerable time to enter, especially for the unskilled typist.

A third major interaction is the entry of urinalysis test results. In this hospital a complete urinalysis requires up to 30 responses; each response may consist of up to six characters. The set of results for each specimen can therefore take two or three minutes to Accepted for publication 4 July 1984 enter by means of the keyboard of the video display terminal. As we may receive over 100 urinalysis requests in a day, over $4 \mathrm{~h}$ of technologist's time is required to enter these results.

In this laboratory the interpretation of creatine kinase and lactate dehydrogenase isoenzyme pat terns is done at the end of each working day. $A$ clinical chemist reviews each pattern and writes a descriptive comment on the worksheet. A technologist then enters these comments (in coded form from a phrase dictionary) into the appropriate patient files. Coded comments considerably reduce the time taken to enter interpretations; however, this procedure requires the technologist to memorise the set of codes corresponding to the required phrases. As there may be up to 100 patterns to process and review in a day, a considerable amount of time and effort is devoted to entry of these interpretations.

We have recently obtained an order and result entry digitising device which interfaces through the video display terminal to the laboratory computer and which considerably speeds the processes of order and result (or interpretative) entry. We describe our experience with this device in comparison with conventional keyboard entry of orders and results.

\section{Material and methods}

COMPUTER INSTALLATION

The computer was an Eclipse MV/6000 32-bit com- 


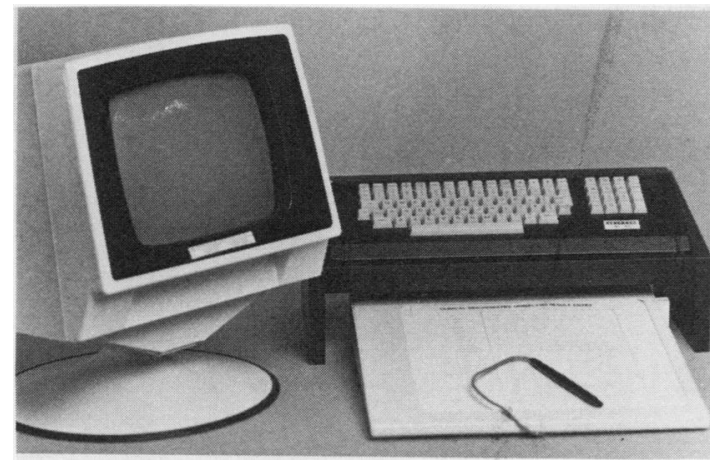

Fig. 1 Configuration of the equipment (studio photograph). The video display terminal is mounted on a stand which allows movement of the screen in all directions to suit the user and the lighting conditions in the room. The keyboard is mounted in a hospital produced bridge which allows ready access to the keyboard while using the digitiser. The digitiser pad is bevelled towards the operator, which allows better visibility of the forms. The stylus cable originates from the top right hand side of the digitiser pad. The form is covered by a thin plexiglass sheet to prevent wear.

puter (Data General (Canada) Ltd, Mississauga, Ontario, Canada) with one megabyte of core memory. We used a Bit Pad Ten digitiser and stylus (Summagraphics Corp, Fairfield, Connecticut, USA) connected to the auxiliary serial port of an XL-87M video display terminal (Cybernex Ltd, Ottawa, Ontario, Canada). The main serial port of the video display terminal was connected to the computer (Fig. 1). Data rates were at 9600 baud. The computer operating and customised programming system were supplied by Medical Information Technology Inc (Cambridge, Massachusetts, USA) written in a dialect of MUMPS.

\section{DIGITISER SET UP}

Order entry, urinalysis, and isoenzyme interpretation result entry forms (Figs. 2-4) were designed and printed in the hospital. The order entry form was designed to be used with the current request form.' The urinalysis result entry form was planned to facilitate sequential entry of the 30 urinalysis responses by matching the sequence of responses on the form to the test sequence appearing on the urinalysis worksheet. The isoenzyme interpretation entry form contained most of the usual interpretative comments in use in this department. The test name, result response, and utility sequences are set up for each form by means of an alignment routine. The top, bottom, left, and right edges of each box on a form are touched with the stylus; these dimensions are stored in a table for each item. In use, touching the box with the stylus anywhere within these predefined boundaries will send the required response to the computer. These alignments are readily verified at suitable intervals.

\section{EXPERIMENTAL PROTOCOL}

Twenty experienced technologists in the department of clinical biochemistry were timed for order entry and urinalysis and isoenzyme interpretation result entry on the conventional (keyboard) and digitiser techniques. We used orders for a range of test requests, a single 30 response urinalysis result, and one short and one long isoenzyme interpretative comment. The Mann-Whitney U test was used to test for the significance of differences between keyboard and digitiser entry time. ${ }^{2}$

\section{Results}

All comparisons between manual (keyboard) and digitised entries showed the superior speed of the digitiser $(p<0.002)$, usually by a factor of from two to fourfold. For example, the fastest entry time for urinalysis results (Fig. 3) by keyboard was $104 \mathrm{~s}$, while the same results when digitised required $40 \mathrm{~s}$ to enter. One long isoenzyme interpretative comment required $9 \mathrm{~s}$ by keyboard but only $3 \mathrm{~s}$ when digitised.

\section{Discussion}

Since this hospital opened in 1972 the annual workload in the department of clinical biochemistry has increased by about $7 \%$ each year to (in 1983) over two million tests annually. There has not been a concomitant increase in staffing; instead, productivity has been increased in two ways. Firstly, microprocessor controlled analysers have been acquired to replace older equipment-for example, a Technicon SMA 12/60 was replaced by the faster, and more easily operated SMA 12/90; individual Beckman electrolyte, glucose, and creatinine analytical modules were replaced by the automatic ASTRA 8 analyser.' This significantly reduced operator time and often allowed a reduction in staffing in particular laboratory areas. Secondly, a laboratory computer system was installed and interfaced to the analytical equipment, thus considerably reducing the time spent on clerical duties-for example, sample labels, worksheet preparation, result logs, and report preparation.

Paradoxically, some of the procedures that we used after installation of the laboratory computer were more time consuming than the manual proce- 


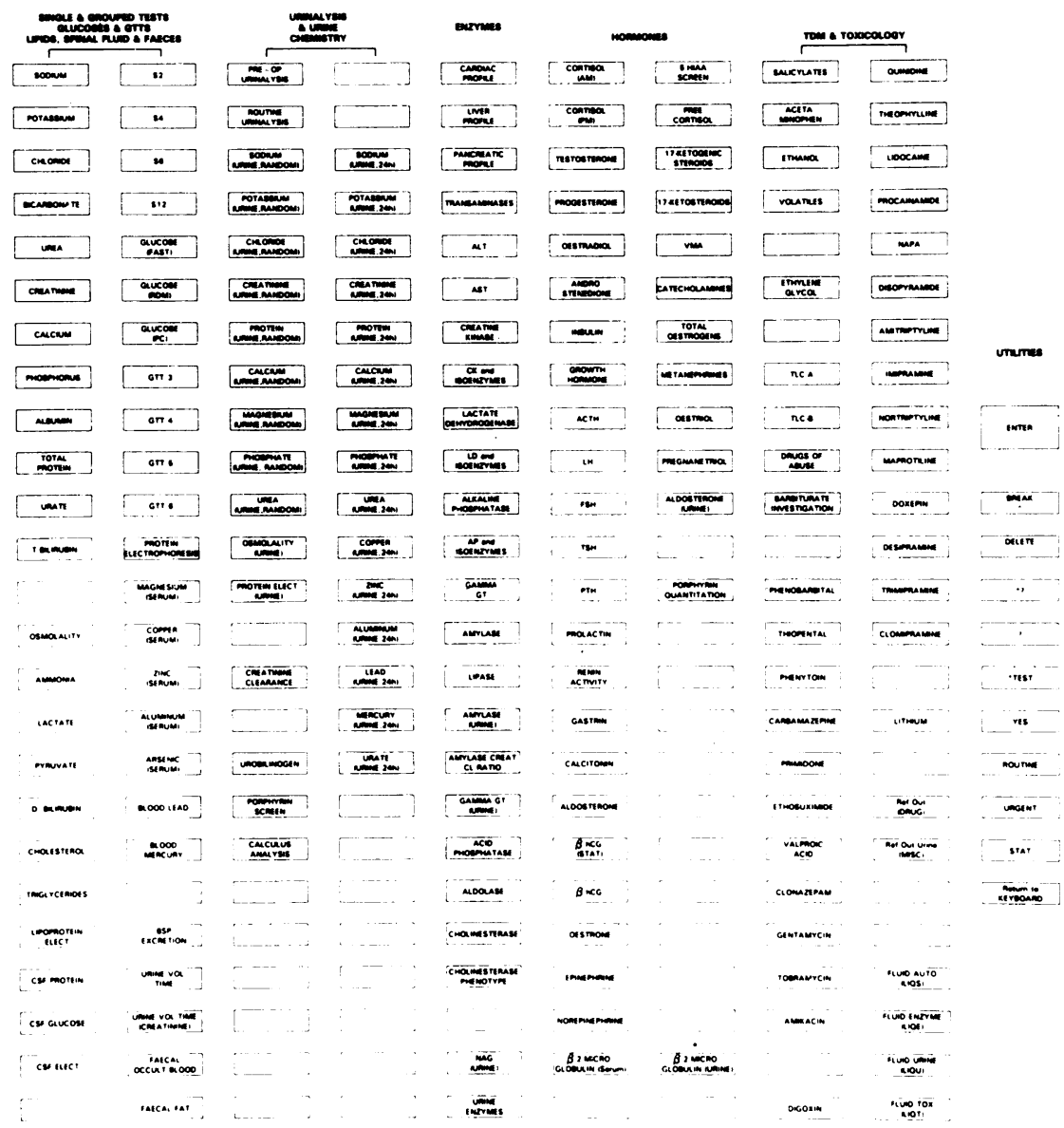

Fig. 2 Order entry form. This form is used on the two digitiser pads in the sample reception area. Each group of tests is described by the header at the top of each column and printed in bold type. The layout roughly follows that used in the clinical biochemistry requisition form. ${ }^{1}$ The column marked UTILITIES (on the right hand side of the form) allows the operator to choose test request options present on the video display screen, correct wrong entries, and to classify test requests as routine, urgent, or stat.

dures that preceded them. For example, before computerisation, urinalysis requests were received (from clinical units) on a two part form. Results were written directly on to this form; the top copy was returned to the unit and the second copy was retained by the laboratory. After computerisation, urinalysis requests were made on the request form, ${ }^{1}$ a computer produced worksheet was printed, and the results were written on the worksheet and then transferred into the computer by manual entry using the keyboard of a video display terminal. The number of responses for a complete urinalysis is considerable (Fig. 3) and their entry takes an appreciable time, especially for the indifferent typist. A similar type of predicament was created by isoenzyme pattern interpretation.

Clearly, there are a number of solutions to our problem of rapid entry of analytical data into a computer file. Many of these solutions require precoding of the results, and reading of these codes by a card reader (for punched cards) or an optical wand for bar $\operatorname{code}^{3}$ or optical character readers. ${ }^{4}$ Alternatively, a predesigned form can be marked in coded areas with soft lead pencil, and this document can be read with an optical mark reader. ${ }^{5}$ A popular solution, available on many laboratory computer systems, is the use of a customised keyboard containing an array of precoded keys. More elaborate solutions 


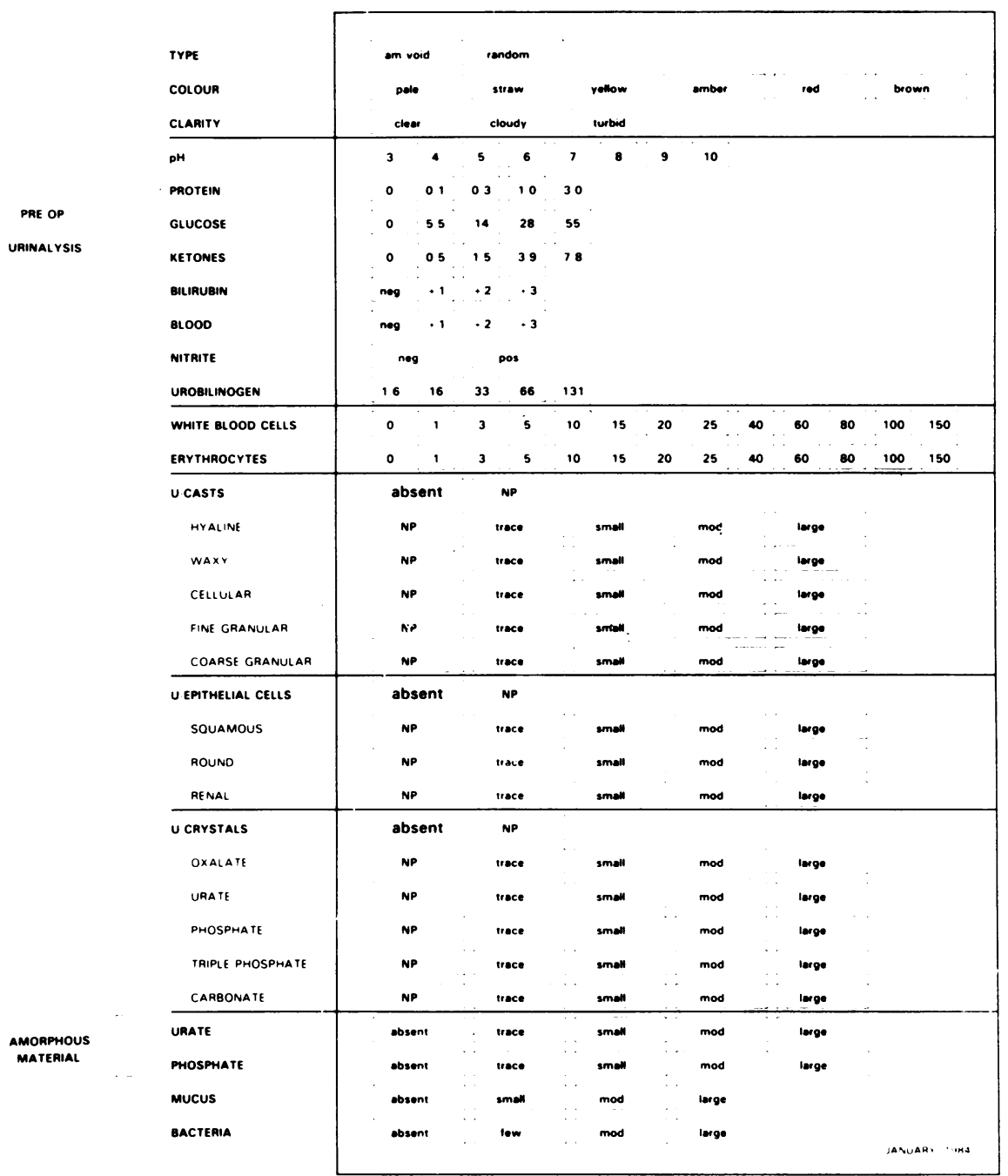

Fig. 3 Urinalysis result entry form. This form is used on the two digitiser pads in the urinalysis laboratory. Two test groupings can be entered from this form. The preop (presurgical) urinalysis consists of the marked tests. A complete urinalysis consists of all tests shown on the form. The responses are structured so that if no casts, epithelial cells, or crystals are present, the tests U-CASTS, U-EPITHELIAL CELLS, or U-CRYSTALS (all shown in bold type) are reported as absent. If, on the other hand, casts are present, NP (for no print) is selected for $U$-CASTS and this test will be replaced by the appropriate response(s) from within the CASTS group, such as $U$-waxy casts mod(erate) and so on. This arrangement prevents the printing of a large number of "absent" responses.

include light pen or finger touch sensitive video display terminal screens, where the available responses are displayed and the options are chosen by touching the appropriate box.

In our view digitisers are an attractive alternative. They are fast, technologically reliable (because of their simplicity), easy to set up and use, and easy to modify while the current system is still in use; and there is a wide range of possible laboratory applications, although we have applied their use to only three operational bottle necks in this laboratory. We therefore believe that digitiser techniques may be a valuable supplementary approach to the problem of data entry. 


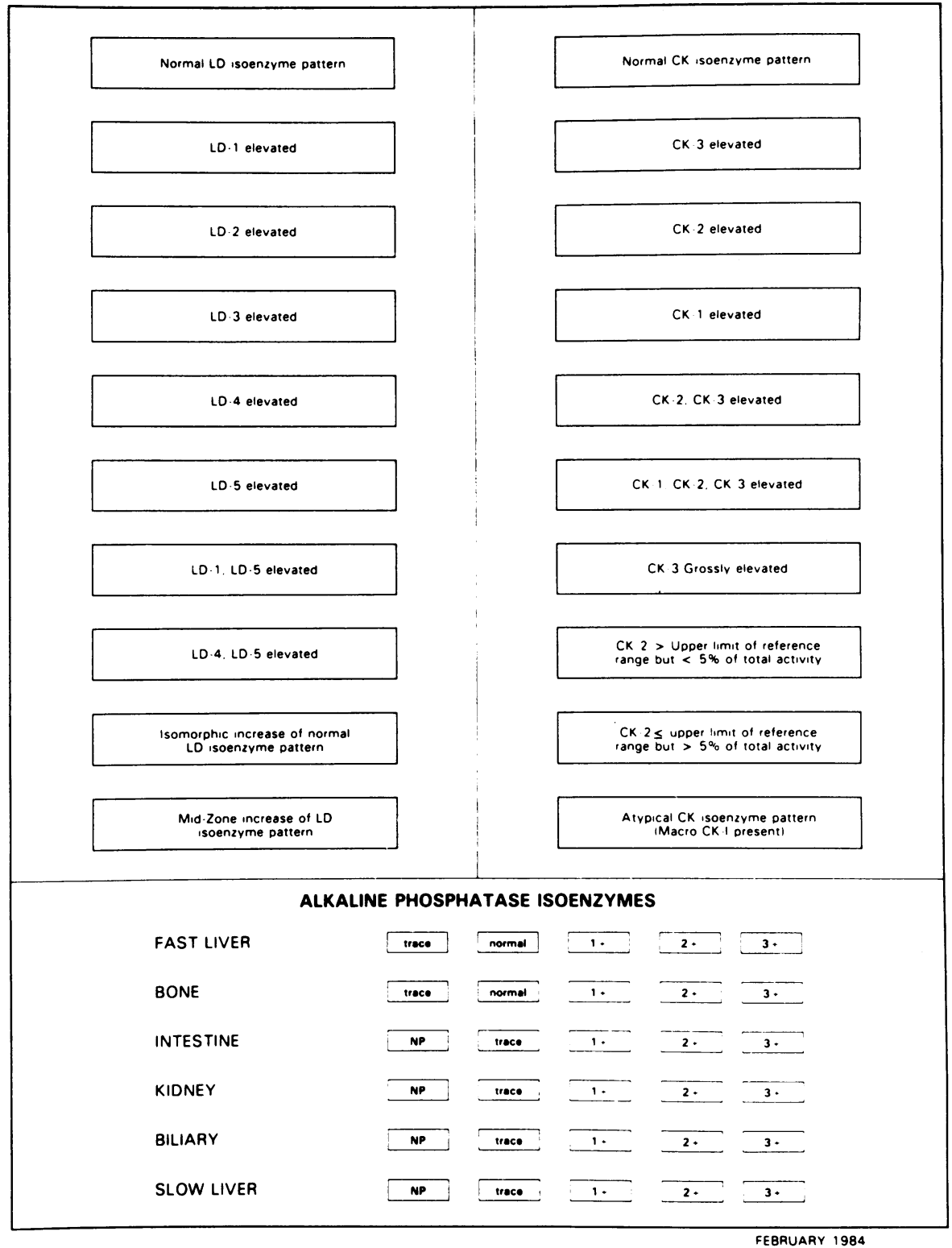

Fig. 4 Isoenzyme interpretation form. This form is used on the digitiser pad in the isoenzyme laboratory. The most common interpretative comments are listed for the $L D$ (lactate dehydrogenase) isoenzymes (left hand side) and for the CK (creatine kinase) isoenzymes (right hand side). In addition, the alkaline phosphatase isoenzyme responses are tabulated at the foot of the form.

\section{References}

' Henderson AR. The test request form: a neglected route for communication between the physician and the clinical chemist? J Clin Pathol 1982;35:986-98.

${ }^{2}$ Siegel S. Nonparametric statistics for the behavioral sciences. New York: McGraw-Hill, 1956:116-27.

${ }^{3}$ Laue D. The barcode-a universal system of identification in the medical laboratory. In: Siemaszko F, ed. Computing in clinical laboratories. Tunbridge Wells: Pitman Medical, 1978:184-90.
4 Dudeck J, Michel H. OCR Readers. In: Siemaszko F, ed. Computing in clinical laboratories. Tunbridge Wells: Pitman Medical, 1978:196-205.

${ }^{5}$ Coleman JC, Linington AW. Optical mark readers in clinical microbiology. In: Computing in clinical laboratory. Tunbridge Wells: Pitman Medical, 1978:191-5.

Requests for reprints to: Dr AR Henderson, Department of Clinical Biochemistry, University Hospital, PO Box 5339, Postal Stn. A, London, Ontario, Canada N6A 5A5. 